\title{
Nontuberculous Mycobacterial Pulmonary Infections
}

\author{
An Expert Interview with Juzar Ali \\ Louisiana State University Health Sciences Center (LSUHSC) School of Medicine, New Orleans, LA, US
}

DOI: https://doi.org/10.17925/USRPD.2018.13.1.17

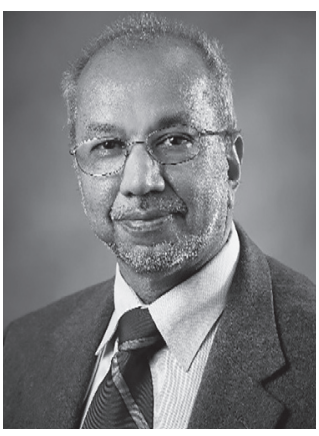

Juzar Ali

Dr Juzar Ali, Professor of Medicine, is an academic clinician/clinical educator and healthcare administrator within the section of pulmonary critical care at Louisiana State University Health Sciences Center (LSUHSC) New Orleans. He has worked nationally and internationally in the field of tuberculosis, and at the LSUHSC has developed programs that contribute to the education of medical students, allied health professionals, and the community. His clinical, academic, and research interests include the diagnosis and management of tuberculous and non-tuberculous mycobacterial disease and related co-morbidities in pulmonary medicine. He has participated in various multicenter studies and trials of tuberculosis and non-tuberculous mycobacteria, and has co-authored a textbook, Pulmonary Pathophysiology, a symptom-based clinical and pathophysiologic review of lung diseases. He has also developed a Chest Radiology PRE-TEST series for medical students and residents. Dr Ali is a recipient of numerous teaching awards over the last two decades. He was recently recognized as a member of the Best Doctors in America and at a national level was awarded the Fulbright Scholar Teaching Grant and Alumni award and Fulbright Senior Scholar Award twice with academic tenures in Turkey and India. He has also been a Visiting Faculty to Vietnam under the US-Vietnam Education Fund Program. Currently his focus is on developing a Mycobacterial Diseases Mentoring and Training program in the section and creating a mycobacterial diseases clinical and basic science registry and database in collaboration with other departments at LSUHSC.

\section{Keywords}

Nontuberculous mycobacteria, mycobacterium avium, amikacin liposomal inhalation suspension

Disclosure: Juzar Ali is a consultant/speaker/ advisory board member for Oxford Immunotech and Insmed. He is a principal investigator in the Convert 212 and 312 Studies and the Willow Study. Juzar Ali is also a member of the journal's editorial board. Review Process: This is an expert interview and, as such, has not undergone the journal's standard peer review process.

Authorship: The named author meets the International Committee of Medical Journal Editors (ICMJE) criteria for authorship of this manuscript, takes responsibility for the integrity of the work as a whole, and has given final approval for the version to be published.

open Access: This article is published under the creative Commons Attribution Non-commercial License, which permits any non-commercial use, distribution, adaptation, and reproduction provided the original author and source are given appropriate credit. (c) The Author 2018.

Received: August 6, 2018

Published Online: September 17, 2018

Citation: US Respiratory \& Pulmonary

Diseases. 2018;3(1):17-8

Corresponding Author: Juzar Ali, LSUHSC, 1901 Perdido Street, Suite 3201 MEB, New Orleans, LA. 70112, US. E: JALI@|suhsc.edu

Support: No funding was received in the publication of this article.
$\mathrm{P}$ ulmonary infections caused by nontuberculous mycobacteria (NTM) are increasing in prevalence, and impose a significant health and economic burden. There are around 150 species of NTM, which are ubiquitous in the environment, and infections are transmitted by environmental spread, particularly from soil and water. In an expert interview, Dr Ali discusses risk factors for and management of NTM infections.

\section{Q. What are the major risk factors for nontuberculous mycobacteria lung infections?}

Over the last few years, the awareness and need for treatment and management of NTM pulmonary disease has been increasing. The worldwide distribution of NTM respiratory disease has now been well documented with Mycobacterium avium pulmonary infection being the predominant pathogen. ${ }^{12}$ The prevalence of this infection in the coastal belt of the US has been reported between 150-200 per 100,000 people, this being $70 \%$ of all NTM cases in the US. In the US it is projected that we will see an $8 \%$ annual increase in its prevalence. ${ }^{3}$ Other than the previously recognized risk factor in cystic fibrosis, HIV, and dual infection seen with tuberculosis, we are now recognizing this infection in chronic obstructive pulmonary disease, pulmonary fibrosis, sarcoidosis, and associated with gastroesophageal reflux disease/naso-sinusitis and primary bronchiectasis. Smoking, low body weight, immunosuppression especially in rheumatological diseases, soil, and environmental exposure remain as associated conditions and risk factors.

\section{Q. What are the most challenging aspects in the diagnosis and treatment of nontuberculous mycobacteria lung infections?}

Criteria of diagnosis of NTM pulmonary infection notwithstanding, NTM infection is a product of the pathogenicity of the mycobacteria, load and duration of infection, and the host response based on underlying and comorbid disease, degree of airway and systemic immunosuppression of the host and other genetic factors. With primary bronchiectasis, the challenge is to understand and manage the "chicken and the egg" paradox. It is still not clear what starts first in the typical young woman with so-called Lady Windermere syndrome. However, it is now well understood that the bronchiectatic type of NTM, especially Mycobacterium avium complex, differs in its course, management and prognosis than that of the fibrocavitary type. Further, $40 \%$ of patients are more likely to die if associated with comorbid conditions as outlined earlier. 
With this in mind, the keys to diagnosis and management lie in understanding the role of the upper airway and lower airways host defense factors; mucociliary clearance; and factors related to defense mechanism at the alveoli and lung parenchymal macrophage, and cytokine level. This coupled with the microbe itself, the serovariability of its colonies, and specific pathogenesis makes the diagnosis and prediction of disease confounding. Survival and immune evasion based on biofilm formation, cell wall characteristics blocking vacuolar acidification, its persistence in an anaerobic intracellular environment, and induction of NTM-related genes that enhance replication further enhances the chronicity of infection and its relapse. The underlying anatomic distortion in the architecture of the lung probably perpetuates the inflammatory and microbiological persistence of infection. In primary bronchiectasis, the unopposed neutrophilic elastase and serine proteinase activation, along with oxygen intermediates and inflammatory cytokines, lead to inflammation, edema, neo-vascularization, and tissue destruction. This occurs both at an airway and cellular cytokine level leading to increased mucus secretions and impaired mucociliary clearance. The sequel to this may then be granuloma, nodule or cavitary formation, which adds chronicity to the process despite treatment.

The treatment of NTM pulmonary disease can be limited by a number of factors, such as the hydrophobicity of NTM with the drugs, barriers associated with cell-wall permeability as seen in $M$. chelonae infection, presence of excessive mucus production as seen in $M$ abscessus, and adaptive resistance due to continued exposure. This is further compounded by variability in accuracy, timeliness, and methods of identification of NTM, and the intrinsic intracellular barrier to treatment.

\section{Q. What is your preferred approach to the management of nontuberculous mycobacteria lung infections?}

The preferred approach is multidisciplinary in nature. Antimicrobial therapy is based on limited in vitro sensitivity data and dictated by minimum inhibitory concentrations. It is targeted either as suppressive treatment or acute aggressive long-term treatment with multiple oral, and if needed, inhaled or parenteral antibiotics.,5 Diseases either related to associated lung disorders or underlying immune suppression in rheumatological diseases must be treated concurrently. Specific attention should be paid to naso-sinusitis, gastroesophageal reflux disease, and esophageal motility issues, if present, with close laboratory collaboration to identify subspecies and obtain the best widened susceptibility data to help modify antimicrobial therapy. Concomitant nutritional support to prevent weight loss is crucial. Education and training on an on-going basis to improve airway clearance using nebulizers/postural drainage/percussive devices with appropriate infection control precautions, if applicable, must be tried; however, these devices are not helpful in all patients. Due to the chronicity of the disease process and the persistence and relapse of infection, on-going surveillance, engagement of the patient and care givers in understanding this disease and its course is crucial. Establishing reasonable expectations of treatment and management outcomes with psychosocial support goes a long way in satisfactory patient-centered management.

\section{Q. What advantages could inhaled drugs offer for nontuberculous mycobacteria infections?}

Hitherto, supported by earlier studies and clinical guidelines, inhaled drugs, especially aminoglycosides, have been used to treat gram-negative infection in bronchiectasis and cystic fibrosis. We now have the opportunity, pending regulatory clearance, to use amikacin liposomal inhalation suspension (ALIS) for inhaled therapy in conjunction with the standard oral regimen and in supplement to IV aminoglycosides. ${ }^{6}$ If approved, ALIS, which is composed of the small biocompatible nanoparticle liposomal amikacin, will allow intracellular delivery of this drug directly at a macrophage level and help reduce mycobacterial colony count with resultant lower serum levels than when amikacin is delivered systemically. This may potentially be more effective and with less systemic toxicity.

\section{Q. What are the most promising treatment options in clinical investigation?}

Increased use of clofazimine, linezolid and bedaquiline as indicated in special cases has been clinically accepted. Specific targeted drugs towards M. abscessus and regimens without rifampin for NTM infection in general are on the horizon. ${ }^{7}$
1. Johnson MM, Odell JA. Nontuberculous mycobacterial pulmonary infections. J Thorac Dis. 2014;6:210-20.

2. Hoefsloot W, van Ingen L, Andrejak $C$, et al. The geographic diversity of nontuberculous mycobacteria isolated from pulmonary samples: an NTM-NET collaborative study. Eur Respir J. 2013;42:1604-13.

3. Adjemian J, Oliver KN, Seitz AE, et al. Prevalence of nontuberculous mycobacterial lung disease in U.S. Medicare beneficiaries. Am J Respir Crit Care Med. 2012;185:881-6.

4. Griffith DE, Aksamit T, Brown-Elliott BA, et al. ATS Mycobacteria Diseases Subcommittee. American Thoracic Society; Infectious Disease Society of America. An official ATS/IDSA statement: diagnosis, treatment, and prevention of nontuberculous mycobacterial diseases. Am J Respir Crit Care Med. 2007;175:367-416

5. Haworth CS, Banks J, Capstick T, et al. British Thoracic Society guidelines for the management of non-tuberculous mycobacterial pulmonary disease (NTM-PD). Thorax. 2017;72(Suppl 2):ii1-64.

6. Oliver KN, Griffith DE, Eagle G, et al. Randomized trial of liposomal amikacin for inhalation in nontuberculous mycobacterial lung disease. Am J Resp Crit Care Med. 2017;195:814-23.

7. Wu ML, Aziz DB, Dartois V, Dick T. NTM drug discovery: status, gaps and the way forward. Drug Discov Today. 2018;23: 1502-19. 